\title{
Development of a Thermodynamic Low Order Model for a Twin Screw Expander with Emphasis on Pulsations in the Inlet Pipe
}

\author{
Iva Papes ${ }^{1}$, Joris Degroote, Jan Vierendeels
}

Department of Flow, Heat and Combustion Mechanics, Ghent University, Belgium

\begin{abstract}
A twin screw expander is a positive displacement machine used in various applications of waste heat recovery. The performance of this machine is influenced by internal leakages, gas pulsations formed in the inlet pipe and the properties of the refrigerant. In this paper a multi-chamber mathematical model of a twin screw expander is presented to predict its performance. From the mass and energy conservation laws, differential equations are derived which are then solved together with the appropriate Equation of State $(\mathrm{EoS})$ in the instantaneous control volumes. In order to calculate the mass flow rates through leakage paths more accurately, flow coefficients used in the converging nozzle model were derived from 3D Computational Fluid Dynamic (CFD) calculation. Due to high gas pulsation levels at the inlet port, a coupling with a 3D CFD inlet pipe model is introduced in order to better predict throttling losses. The maximal deviation between predictions by the developed model and 3D CFD calculations of the complete machine is around $5 \%$ for the mass flow rate and the power output.
\end{abstract}

Keywords: Twin screw expander, Thermodynamic model, Multi-chamber, Inlet pulsations, Aungier Redlich-Kwong EoS, Organic Rankine Cycle

\section{Nomenclature}

\section{Symbols}

$\dot{m} \quad$ Mass flow rate $(\mathrm{kg} / \mathrm{s})$

$\dot{Q} \quad$ Heat transfer rate $(W)$

${ }_{5} \rho \quad$ Density $\left(\mathrm{kg} / \mathrm{m}^{3}\right)$

A Area of the leakage path/inlet area $\left(m^{2}\right)$

C Flow coefficient (-)

E Energy $(J / k g)$

$h \quad$ Specific enthalpy $(J / k g)$

$10 \quad k$

$m$

$n \quad$ Rotational speed $(\mathrm{rpm})$

$P \quad$ Power $(W)$

$T \quad$ Temperature $(K)$

$15 \quad u$
$V \quad$ Volume $\left(\mathrm{m}^{3}\right)$

W Work $(J)$

$z \quad$ Number of lobes (-)

\section{Subscripts}

${ }_{20}$ ch Chamber

down Downstream

$i \quad$ Index of the boundary of the working chamber

in Indicated

up Upstream

25 Abbreviations
$A R K \quad$ Aungier Redlich-Kwong
CFD Computational Fluid Dynamics
EoS Equation of State
ORC Organic Rankine Cycle

${ }^{1}$ Corresponding author.Tel:+32(0)92643300,E-mail address:iva.papes@ugent.be Preprint submitted to Journal of Applied Thermal Engineering 


\section{Introduction}

In recent years, one of the leading technologies for waste heat recovery is the Organic Rankine Cycle (ORC). The expander's performance is the crucial factor for achieving a sufficient thermal efficiency in the ORC. For a small scale ORC system, positive displacement machines are highly suitable [1, 2]. In particular, screw expanders show a promising energy conversion [3].

Modelling of a twin screw expander is a complex process and the performance may be calculated using either zero or multidimensional mathematical models. Both models can represent one or more chambers existing within the machine. Zero-dimensional models for twin screw compressors are in use for decades already [4, 5, 6]. In 2007, Janicki developed a simulation program for screw machines which made a distinction between the different working chambers and the connections between them [7] and gave a detailed description how the model is developed. In 2011,

40 Bell presented a mathematical model for a liquid flooded scroll compressor [8]. Despite their speed and relatively accurate results, these models neglect some important transient behaviour inside the suction and discharge ports.

Stosic et al. [9] and Huster [10] developed one-dimensional models for the suction and discharge port coupled with a zero-dimensional model of the working chambers. These models can predict the pressure pulsations in the suction or discharge ports well. However, they do not take into account the shape of the ports but only the change in 4 the cross sectional area and this may change the flow losses in the ports.

A three-dimensional simulation of both suction and discharge ports and working chambers presents the most precise but also computational costly model. One of the additional challenges in three-dimensional calculations is grid generation and grid motion. Until now, only few grid generators have been presented that are able to deliver high quality (block-structured) grids [11, 12]. Consequently, low order models are very valuable for performance 50 prediction of screw machines.

The first analytical model for dry screw expanders was presented in [13]. More recently, the numerical and experimental study of an oil injected twin screw expander for both air and R113 has been presented in [14]. The mathematical model was verified with experiments. The flow coefficients used in the leakage models were constant and were obtained from the experimental results. However, a limitation of the experiments is that they cannot accurately measure flow properties in the clearance area or during the filling.

A typical leakage model used when modelling scroll or screw types of positive displacement machines, is the isentropic converging nozzle [13, 15, 16]. The drawback of this model is that it does not take friction into account. Therefore empirical correction factors are used to compensate for losses due to frictional effects. Another leakage model presented in [17] is kind of Fanno flow. This model includes the viscous effects and it assumes that the leakage

6o passage is a constant area channel. In [18] a hybrid leakage model is presented where an empirical frictional correction factor for the isentropic nozzle model is derived by calculating the mass flow through variable area leakage paths with real gas properties and then it is correlated to the prediction of an isentropic nozzle model. This hybrid model can only be used when the flow is not choked.

The aim of this paper is to present how to model the inlet pulsation and how to derive leakage coefficients using 65 CFD calculations. For this the authors use the results of 3D CFD analysis of the twin screw expander presented in [19]. A similar attempt was presented in [20] where a simplification has been made using the representative geometry of the tip clearance but the comparison with full 3D CFD results is missing. A multi-chamber mathematical model of the same geometry used in CFD calculations is presented and validated by comparing different parameters depending on the rotational angle of the male rotor. The drawback of the multi-chamber model is that it does not include dynamics

70 and losses in the inlet port. Recently the authors presented [21] a multi-chamber model with the results of 3D analysis in the inlet pipe applied as boundary condition. By contrast, the 3D analysis in the inlet pipe and the multi-chamber model are truly coupled in this work, taking into account the mutual interaction. The results of the coupled low order model were compared to the full 3D CFD result. The comparison of the coupled model and the full 3D CFD calculations of the complete machine were introduced for the first time in the literature.

\section{2. Geometric characteristics}

Screw expanders are positive displacement machines with a defined expansion volume ratio. The geometry of the twin screw expander analysed in this paper is shown in figure 1 The configuration of the rotor lobes is $4 / 6$ 


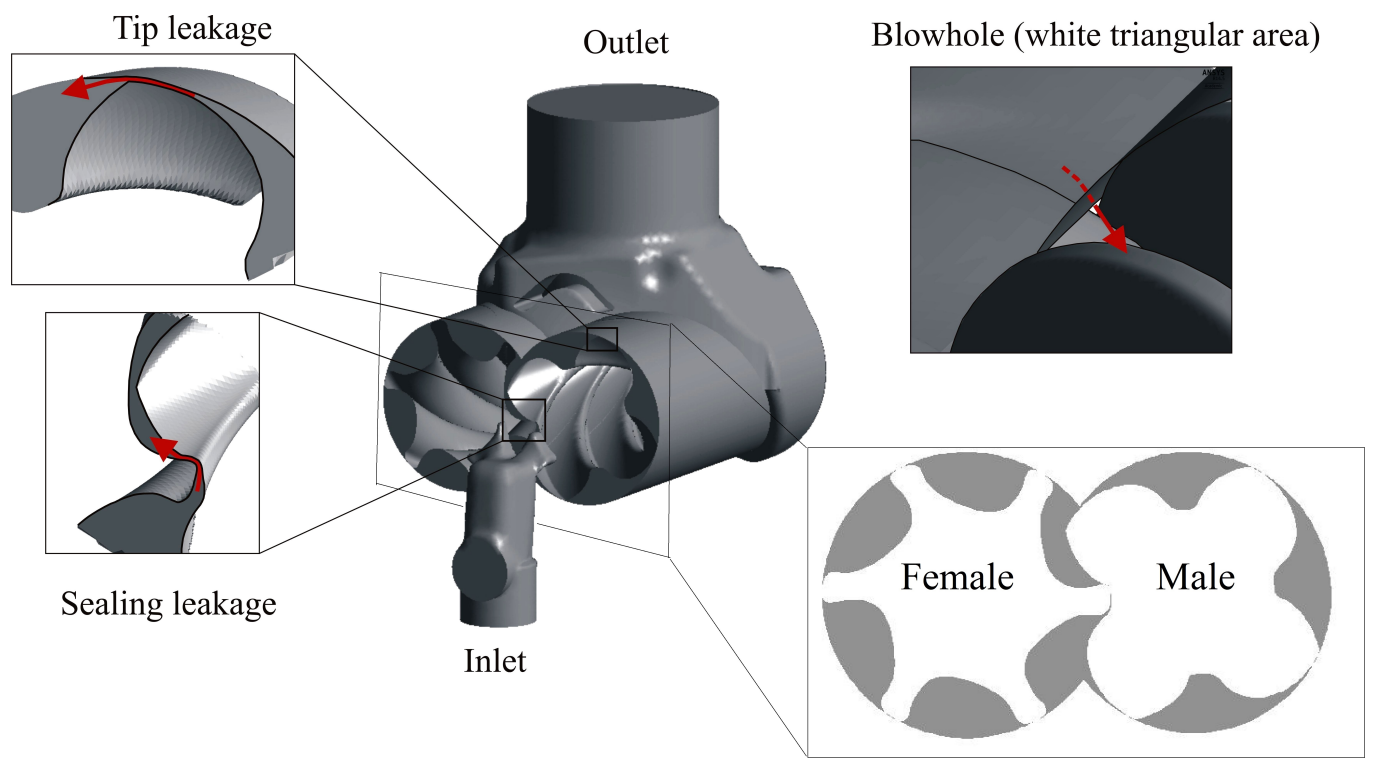

Figure 1: Geometry of the twin screw expander with different leakage flow types

(male/female). The outer diameter of the male and female rotors is approximately $70 \mathrm{~mm}$ with an L/D ratio of 1.9. In order to simulate the expansion process of a twin screw expander, the volume curve and the areas of the leakage paths

The volume of a working chamber as a function of the rotation angle is presented in figure 2a The formation of the chamber starts at $\theta=0^{\circ}$ in figure 2a. Between $\theta=7^{\circ}$ and $\theta=126^{\circ}$, the chamber is in connection with the inlet port. At the beginning the volume of the chamber is rising together with the increase in the inlet surface area, and the chamber is filled with the working fluid. After that the inlet area will start to decrease and the volume of the chamber will continue to increase which results in pre-expansion. Once the inlet port is closed, the working fluid starts to expand with increasing volume of the chamber. At $\theta=387^{\circ}$, the working chamber is connected to the outlet and the working fluid is discharged through the outlet port.

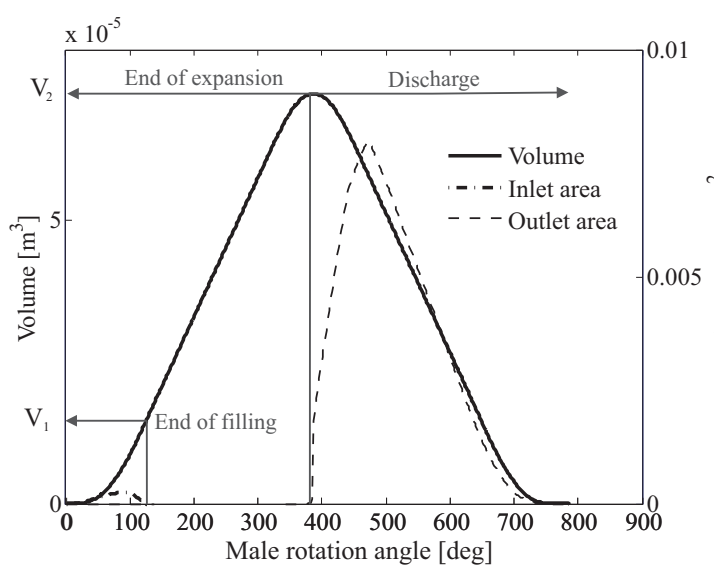

(a) Volume, inlet and outlet curves

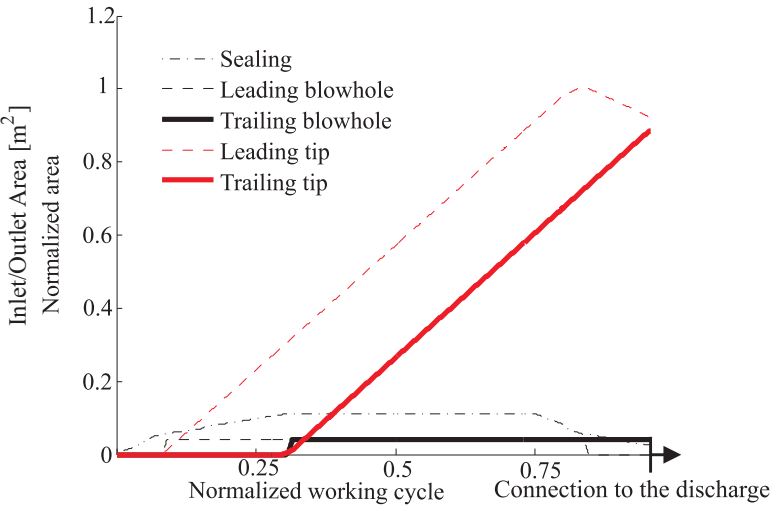

(b) Areas of the leakage paths

Figure 2: Geometrical inputs for the multi-chamber model 
Mass flow rates through the clearances that are forming leakage paths have a significant effect on the performance of screw machines. Three different clearance gaps were evaluated in the CFD analysis performed by the authors

90 mathematical model and are shown in figure 1 Leakages are characterized by the area of the clearance as shown in figure $2 \mathrm{~b}$ Additionally, the leakage flows are distinguished based on the direction of the flow. The gain leakage flow is associated with the flow coming in the chamber from the neighbouring chamber on a higher pressure. Contrary, the loss leakage flow is going from the chamber with higher pressure to the chamber with a lower pressure. Both gain and

\section{Thermodynamic multi-chamber model}

For this model it is assumed that spatial gradients in the important state variables are negligibly small within the working chamber (zero-dimensional model) and thus the fluid is considered to be homogeneous in each chamber. In comparison to the one-chamber model, the multi-chamber model used in this study increases the quality of the simulation results because it introduces the leakage flows between the chambers.

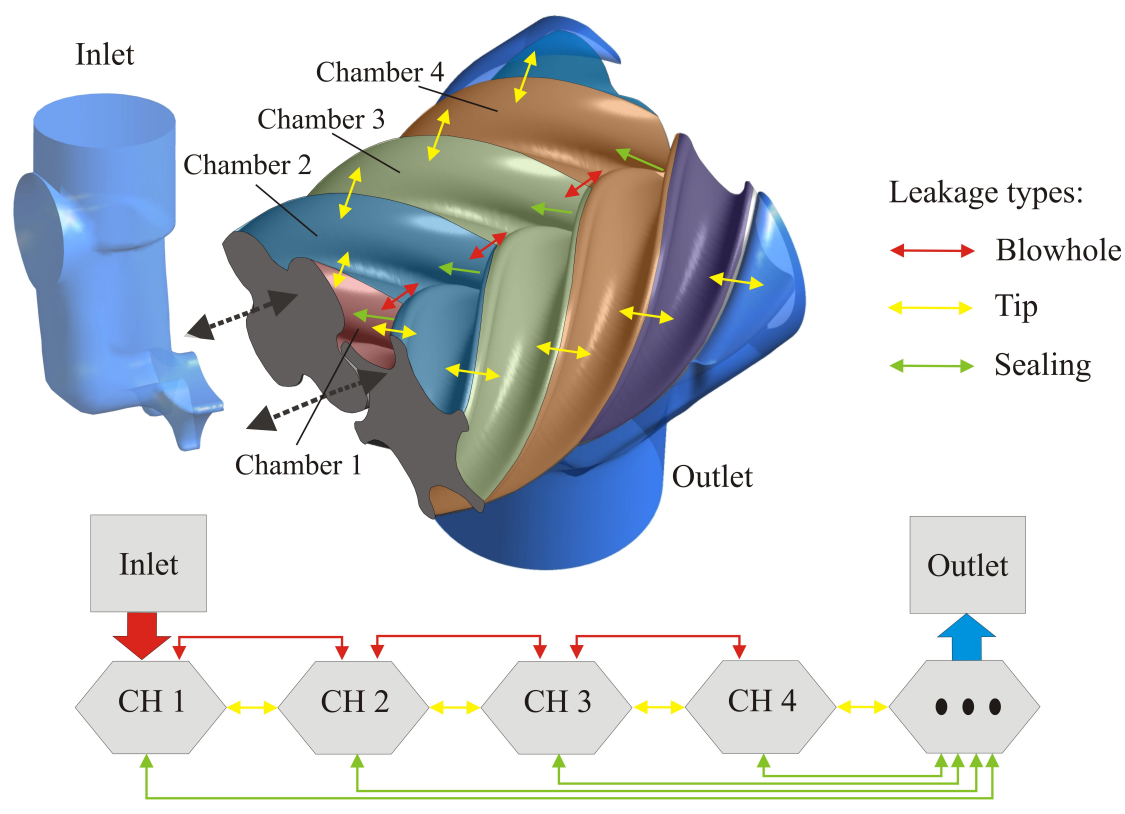

Figure 3: Principle of the connections in the multi-chamber mode

The further explained mathematical model can be used for different geometries of the twin screw expander and for a wide range of operating conditions.

In the analysis that follows, the following assumptions have been employed:

- Potential and kinetic energy of the working fluid in the chambers are negligible.

- The heat transfer between the working fluid and the rotor and between the casing and the ambient is not included in the model, since it was also not included in the CFD model in [19]. This simplification resulted in good agreement between CFD and experiments in [22] for a compressor.

- The working fluid in the chambers can leak through different clearances inside the twin screw expander during the rotation. These leakage flows together with the flow through the inlet port are assumed to pass through a converging nozzle. 
- If the chamber is connected to the outlet port, the pressure in the working chamber becomes equal to the discharge pressure.This assumption will affect the results (since it assumes that the pressure in the outlet port is constant) but this influence is small since the pressure in the outlet port is not affected by high pressure pulsations as is the case in the inlet port shown by CFD calculations in [19].

- Mechanical losses of the screw expander are not included in the model.

- Leakage flows through the end planes are not included in the model. They are also not present in the CFD calculations.

Figure 3 presents the build up of the mathematical model from the chambers, connections between the chambers and connections between the inlet and outlet area. During the filling or discharge, the chamber is in connection with respectively the inlet or outlet pipe. The tip and the blowhole clearances create connections between adjacent chambers while the sealing clearance creates a connection with the chamber that is in connection with the outlet pipe. Depending on the rotational angle of the rotors the appearance of the connections is changing. Therefore the exchange of the mass and energy will happen only through the existing connections for every rotational position of the rotors. The direction of this exchange depends on the working conditions and the properties of the fluid.

\subsection{Conservation laws applied on the working chambers}

A working chamber of a twin screw expander is an open thermodynamic system for which the differential equations of conservation of mass and energy are written. The following forms have been employed in the model:

Conservation of Mass

$$
\frac{d m_{c h}}{d t}=\sum_{i} \dot{m}_{i}
$$

where $\dot{m}_{i}$ represents the mass flow rate coming from all interacting flow paths (boundaries) $i$ into the chamber.

$$
\frac{d E_{c h}}{d t}=m_{c h} \frac{d u_{c h}}{d t}+u_{c h} \frac{d m_{c h}}{d t}
$$

which assumes that the potential and kinetic energies of the mass in the chamber are negligible. As shown in [8], the internal energy of the fluid in the chamber is expanded as a function of $T$ and $v$

$$
d u_{c h}=\frac{\partial u_{c h}}{\partial T_{c h}} d T_{c h}+\frac{\partial u_{c h}}{\partial v_{c h}} d v_{c h}
$$

This can be simplified by using the thermodynamic relation

$$
\frac{\partial u_{c h}}{\partial T_{c h}}=c_{v}
$$

and the relation by Moran and Shapiro [23]

$$
\frac{\partial u_{c h}}{\partial v_{c h}}=\left[T_{c h}\left(\frac{\partial p_{c h}}{\partial T_{c h}}\right)_{v_{c h}}-p_{c h}\right]
$$

which result in

$$
d u_{c h}=c_{v} d T_{c h}+\left[T_{c h}\left(\frac{\partial p_{c h}}{\partial T_{c h}}\right)_{v_{c h}}-p_{c h}\right] d v_{c h} .
$$


Consequently, the overall conservation of energy can be expressed as

$$
\frac{d E_{c h}}{d t}=-p_{c h} \frac{d V_{c h}}{d t}+\sum_{i} \dot{m}_{i} h_{i}+\dot{Q}_{c h}
$$

The right hand side of the equation consists of the following terms:

- $p \frac{d V}{d t}$ is the thermodynamic work supplied to the rotors during the expansion.

- $\sum \dot{m}_{i} h_{i}$ is the total energy gain due to gas inflow and outflow from the chamber. The enthalpy $h_{i}$ is the enthalpy of the upstream state point of the flow path. This means that if the flow is out of the chamber, the enthalpy is that of the chamber and if the flow is into the chamber then the enthalpy is that of the chamber from which the flow is going into the chamber. The summation of the product $\dot{m}_{i} h_{i}$ is done for all interacting flow paths (boundaries) $i$ of the chamber.

- $\dot{Q}_{c h}$ term presents the heat exchange between the fluid and the expander screw rotors and casing and through them to the surrounding.

If the process is assumed to be adiabatic $(\dot{Q}=0)$, using eq. 2 and eq. 7 yields the equation

$$
m_{c h} \frac{d u_{c h}}{d t}+u_{c h} \frac{d m_{c h}}{d t}=-p_{c h} \frac{d V_{c h}}{d t}+\sum_{i} \dot{m}_{i} h_{i}
$$

The left hand side of this equation can be expanded using eq. 6and the thermodynamic relation $u=h-p v$ :

$$
m_{c h} c_{v} \frac{d T_{c h}}{d t}+m_{c h}\left[T_{c h}\left(\frac{\partial p_{c h}}{\partial T_{c h}}\right)_{v}-p_{c h}\right] \frac{d v_{c h}}{d t}+\frac{d m_{c h}}{d t}(h-p v)
$$

If the derivative of the volume is expressed as

$$
\frac{d V_{c h}}{d t}=m_{c h} \frac{d v_{c h}}{d t}+v \frac{d m_{c h}}{d t}
$$

and is used in eq. 9 together with the right hand side of eq. 8 , this yields

$$
m_{c h} c_{v} \frac{d T_{c h}}{d t}+T_{c h}\left(\frac{\partial p_{c h}}{\partial T_{c h}}\right)_{v}\left[\frac{d V_{c h}}{d t}-v_{c h} \frac{d m_{c h}}{d t}\right]+h_{c h} \frac{d m_{c h}}{d t}=\sum_{i} \dot{m}_{i} h_{i}
$$

In the end, the following equation for the derivative of temperature with respect to time is obtained [8]

$$
\frac{d T_{c h}}{d t}=\frac{-T_{c h}\left(\frac{\partial p_{c h}}{\partial T_{c h}}\right)_{v}\left[\frac{d V_{c h}}{d t}-v_{c h} \frac{d m_{c h}}{d t}\right]-h_{c h} \frac{d m_{c h}}{d t}+\sum_{i} \dot{m}_{i} h_{i}}{m_{c h} c_{v}} .
$$

The thermodynamic properties of the working fluid R245fa are retrieved using the Aungier Redlich-Kwong EoS [24]. The validation of the Aungier Redlich-Kwong EoS for the range of operating conditions used in this study is presented in [19]. 


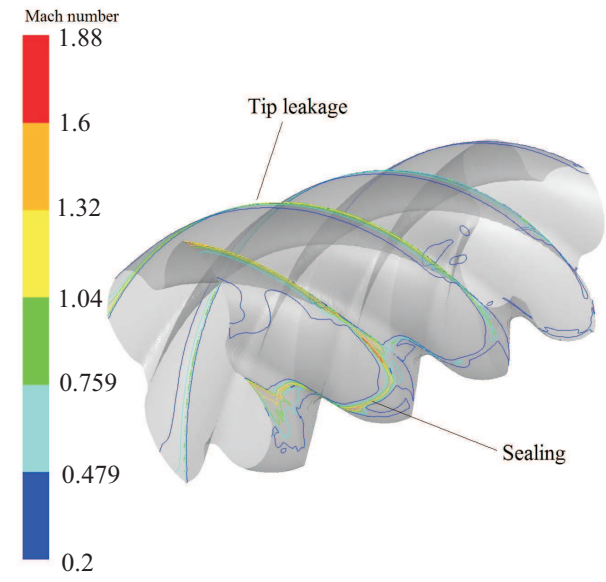

(a) Male rotor

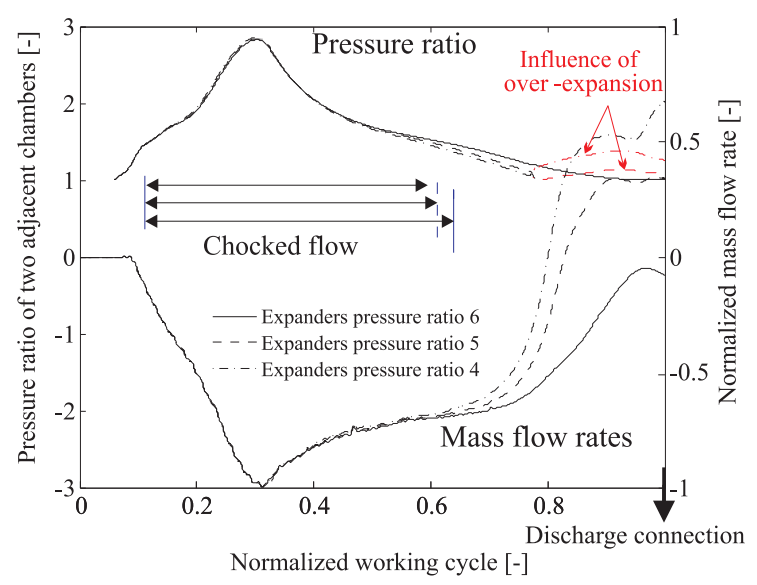

(b) Leading tip leakage

Figure 4: Mach numbers in leakage flows area extracted from the CFD analysis presented in [19] for three different pressure ratios: a) colors from yellow to red indicate choked flow b) during filling and expansion both pressure ratio and mass flow rate between two chambers forming the leading tip leakage are shown. The duration of the choking is also indicated.

\subsection{Leakage analysis}

Leakage flows in a twin screw expander play an important role in the total flow rate and are dependent on the area of the leakage gap, the properties of the refrigerant and the working conditions. The typical model for leakage flows is the converging nozzle. This model considers for choking when the Mach number reaches 1.

In figure $4 \mathrm{a}$ the distribution of the Mach number through the tip and sealing clearance is shown. It can be seen that the flow in these leakage paths is choked during the filling and expansion. The flow through the inlet area is on the contrary never choked. This supports the usage of the converging nozzle model which accounts for both choked and non-choked conditions. Additionally, the pressure ratio between chambers that are forming the tip leakage path and the mass flow rate through it for different expander pressure ratios are shown in figure $4 \mathrm{~b}$. Negative mass flow rate corresponds to mass flow leaving the chamber. Over-expansion occurs if the pressure ratio of the expander is 4 or 5. Since the pressure in the chamber is then lower than the discharge pressure, the mass flow going through the tip leakage becomes positive.

The mass flow through the leakage paths and the inlet port using the converging nozzle model [8] is calculated as follows:

$$
\dot{m}_{\text {nozzle }}=C A \sqrt{p_{\text {up }} \rho_{\text {up }}} \sqrt{\frac{2 k}{k-1}\left(p_{\text {ratio }}^{2 / k}-p_{\text {ratio }}^{(k+1) / k}\right)}
$$

where the function of pressure ratio is defined as:

$$
p_{\text {ratio }}= \begin{cases}\left(1+\frac{k-1}{2}\right)^{k /(1-k)} & p_{\text {down }} / p_{\text {up }} \leq\left(1+\frac{(k-1)}{2}\right)^{k /(1-k)} \\ p_{\text {down }} / p_{\text {up }} & p_{\text {down }} / p_{\text {up }}>\left(1+\frac{(k-1)}{2}\right)^{k /(1-k)}\end{cases}
$$

These mass flow rates are then used in the integration of the mass and temperature differential equations.

One of the focuses in this work was extracting the flow coefficients C from the 3D CFD calculations. For every angular position the following parameters were stored during the 3D CFD simulations of the complete expander:

- area of the leakage path of interest 
- pressure ratio between the chambers that are forming the leakage path of interest

- mass flow rate through the leakage path of interest

- pressure, temperature and specific heat ratio of the chamber with higher pressure

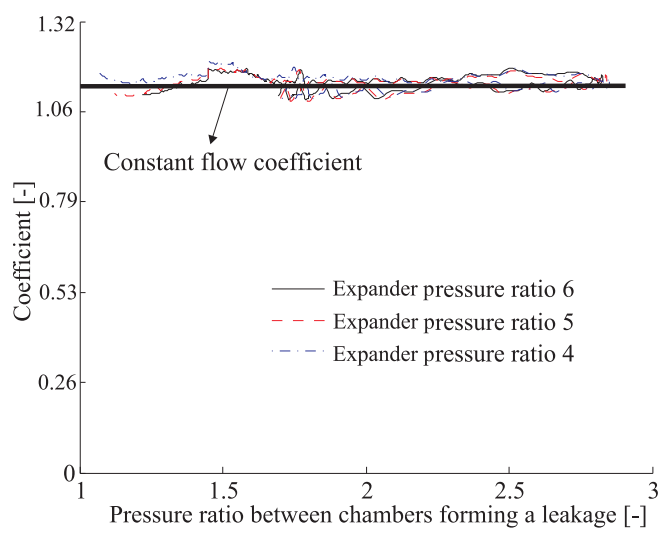

Figure 5: Flow coefficient through the tip clearance extracted from the 3D CFD analysis presented in [19]. The derived constant flow coefficient used in the multi-chamber low order model is indicated. The coefficient is scaled with an arbitrary factor chosen larger then 1.

As a result, the flow coefficients $\mathrm{C}$ were derived for different leakage paths which are then used in the developed multi-chamber model. An example of the calculated flow coefficient for the tip leakage path is shown in figure 5. The flow coefficient is show with respect to the pressure ratio between the chambers that are forming that leakage path. From these results, a constant flow coefficient is calculated and used in the developed multi-chamber model).

\section{Model of the inlet pipe for prediction of the gas pulsations}

Pulsations in the inlet pipe play an important role in the pressure difference between the chamber and the inlet pipe which influences the mass flow rate during the filling. These pulsations can be calculated with 3D CFD calculations as is done in this work or with a one-dimensional model as in [9, 10]. The pressure pulsations from 3D CFD analysis of the entire machine are shown in figure 6 (right).
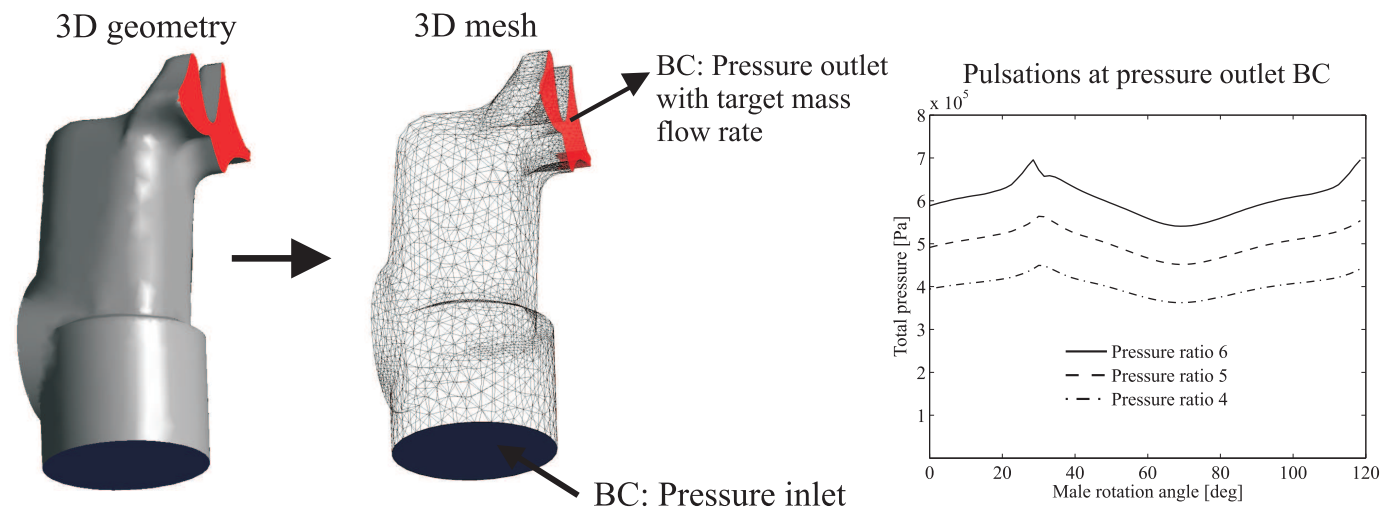

Figure 6: Left: Geometry and the mesh of the inlet pipe. Right: Pressure pulsations in the interface plane of the inlet port (results from CFD analysis)

The authors combined the 3D CFD model of the inlet pipe with the multi-chamber model described in the previous section. This coupled model requires much less time than a complete 3D CFD analysis and still obtains sufficient accuracy, as will be explained below. 
The flow calculation in the 3D inlet model is carried out by means of ANSYS Fluent. Therefore a numerical grid was provided as shown in figure 6 (middle). The time step size corresponds to the time required for a rotation of $1^{\circ}$ of male rotor and it is dependent on the expander's rotational speed. The output of the simulation program is the pressure field at the interface between the inlet pipe and the chamber which is changing depending on the position of the lobes in the multi-chamber model.

\section{Solution Method}

In this section the solution method of the multi-chamber model together with the coupling between the multichamber and the 3D inlet model is presented. As seen in Section 3.1., through the use of conservation of mass and energy, a system of differential equations for mass and temperature can be obtained in each chamber. The system of differential equations is solved by using the forward Euler method applied on all chambers. Once the geometrical inputs (volume change and leakage areas) have been provided to the model, the temperature and pressure of the chambers at the starting angle are initialized with guess values. Then for each time step, the mass flow rates going in or out of the chambers are calculated and the temperature in the next step is obtained with the forward Euler method. Since the mass, the volume and the temperature are then known, the pressure and the density can be updated. In order to ensure the stability and the accuracy of the calculations the number of time steps per cycle can be increased within the multi-chamber model.

The coupling of the multi-chamber and 3D inlet model requires the transfer of data and information between the two models. The flowchart of the method of execution and coupling between the models is shown in figure 7 . As seen in figure 7, two time-dependent informations are exchanged between the multi-chamber and the 3D inlet model: (1) Output of the multi-chamber model: target mass flow rate used in the pressure outlet boundary condition in the 3D inlet model. (2) Output of the 3D inlet model: pressure pulsations at the end of the inlet pipe used in the multi-chamber model. Both informations are written in text files, mass.txt and pressure.txt respectively. The solution of coupling the multi-chamber model and the 3D inlet model has to become periodic and converged. The convergence criterion is determined as the normalised root mean square difference between two iteration steps of pressures as a function of rotational angle and is set to 0.01 . The coupling of the $3 \mathrm{D}$ inlet pipe simulation with the multi-chamber model is further called the coupled model. The convergence of the pressure within the working chamber of the coupled model is shown in Section 6.1.

\subsection{Performance parameters}

The solution of the coupled model allows the calculation of the performance parameters like average mass flow rate and indicated power. The indicated work of a twin screw expander can be expressed as the area of the indicated P-V diagram:

$$
W_{\text {ind,cycle }}=\int_{\text {cycle }} V d p
$$

It represents the indicated work transferred to the male and female screw rotors during the filling, expansion and discharge processes. The indicated power of the twin screw expander can then be calculated using the indicated work as:

$$
P_{\text {ind }}=\frac{W_{\text {ind,cycle }} z n}{60}
$$

with $\mathrm{z}$ the number of male lobes and $\mathrm{n}$ the rotational speed in $\mathrm{rpm}$.

\section{Results and Discussion}

\subsection{Performance parameters}

In Table 1, comparison of power and mass flow rate between the developed coupled model and the full CFD model are presented. The comparison has been made for pressure ratios $\pi=6,5$ and 4 and for rotational speeds of $6000 \mathrm{rpm}$, $4000 \mathrm{rpm}$ and $8000 \mathrm{rpm}$. It can be seen that the maximal deviation is around $5 \%$ for power and mass flow rate. 


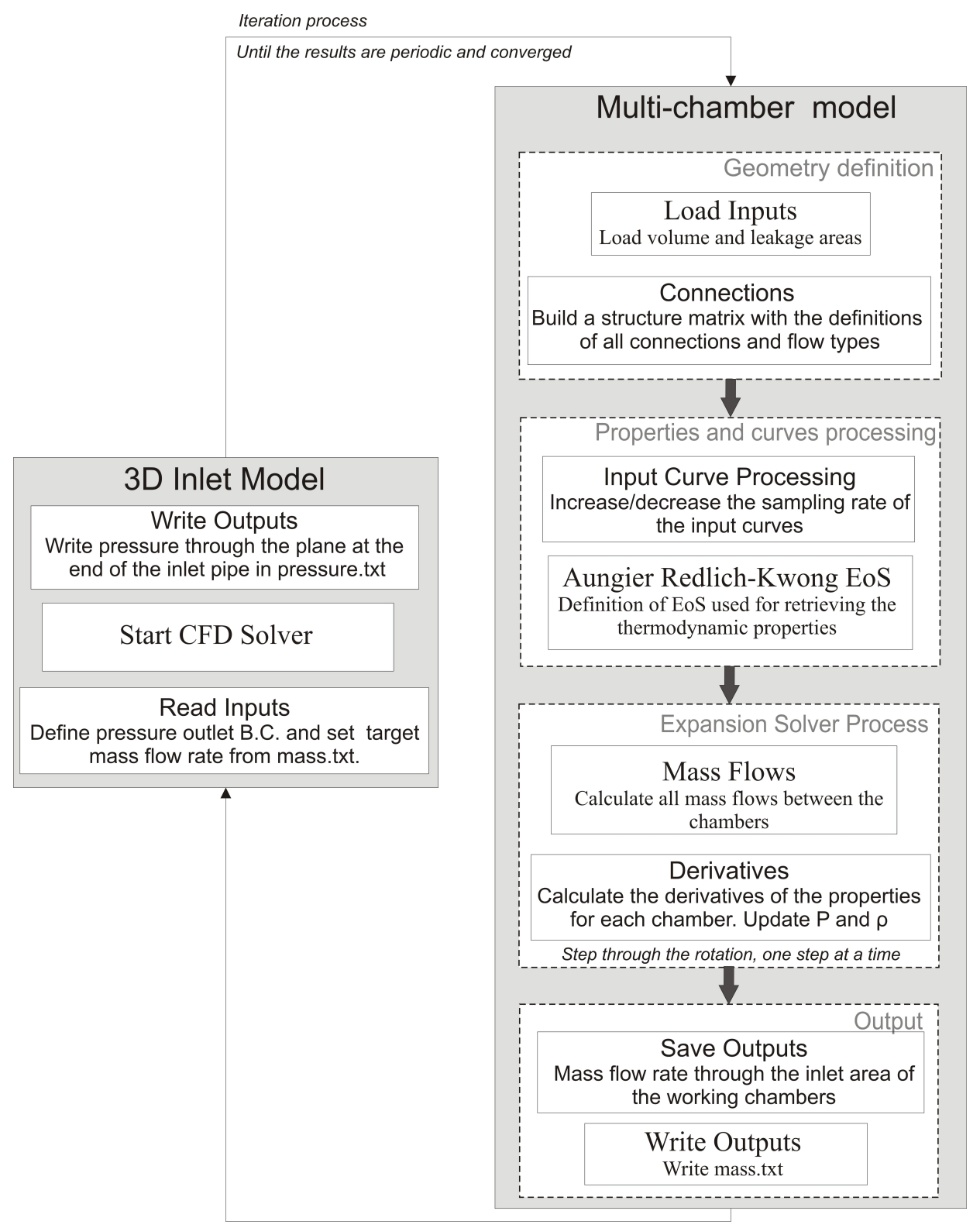

Figure 7: Flowchart of method execution

\subsection{Convergence of the solution}

In this section the convergence of the pressure is presented. In figure 8 different iteration steps are shown in order to highlight the importance of coupling the multi-chamber and the $3 \mathrm{D}$ inlet model. In the first iteration step the pressure in the inlet pipe is set as constant as a function of time and is used in the multi-chamber model. Although the constant pressure in the inlet pipe is used in some currently applied models, there is a big difference with the CFD results for the filling phase (red circle in figure 8). As the number of iterations of the coupled model is increasing the pressure difference between the coupled model and the full 3D CFD model is decreasing. It can be concluded that the most important factor during the filling phase is the difference between the pressures in the working chamber and the inlet pipe. However this difference is greatly affected by the gas pulsations in the inlet pipe which are in this paper modelled using a 3D CFD inlet model.

The difference in computing time and hardware requirement for a full 3D CFD model, a coupled 3D inlet pipe 


\begin{tabular}{|l|c|c|c|c|c|}
\hline Case number & 1 & 2 & 3 & 4 & 5 \\
\hline Expander's pressure ratio & 6 & 5 & 4 & 6 & 6 \\
\hline Speed [rpm] & 6000 & 6000 & 6000 & 4000 & 8000 \\
\hline Power [kW] & 4.72 & 3.46 & 2.18 & 3.18 & 5.11 \\
\hline Full 3D CFD model & 4.68 & 3.44 & 2.17 & 3.26 & 5.36 \\
\hline Developed coupled model & 0.85 & 0.58 & 0.46 & 2.45 & 4.6 \\
\hline Deviation between models [\%] & | & \multicolumn{7}{|l|}{} \\
\hline Mass flow rate [kg/s] & 0.1469 & 0.121 & 0.095 & 0.1055 & 0.1618 \\
\hline Full 3D CFD model & 0.143 & 0.117 & 0.094 & 0.1055 & 0.164 \\
\hline Developed coupled model & 2.65 & 3.3 & 1.05 & 0 & 1.34 \\
\hline Deviation between models [\%] & 2.65
\end{tabular}

Table 1: Results for mass flow rates and power outputs for the developed coupled model and the full CFD model

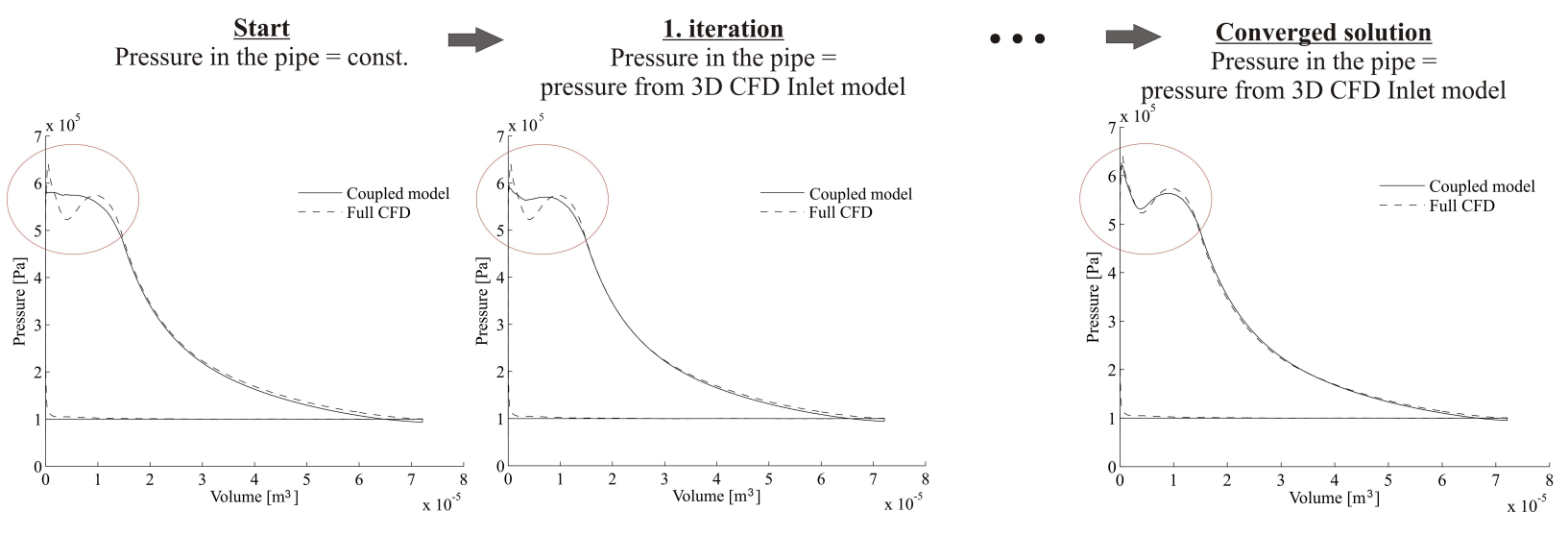

Figure 8: Convergence of the pressure in the developed coupled model

model with a multi-chamber model and a multi-chamber model without inlet pipe model is shown in figure 9 . It can be seen that for a converged and periodic solution in a full 3D CFD model 336 hours are necessary. For a coupled model (on average 8 iterations between the 3D inlet pipe model and multi-chamber model) the total used time is 14 hours. In a case of multi-chamber model, only one minute is necessary on a personal computer.

In figure 8 (left side), at the start of the coupling, a result of a multi-chamber model not coupled with the 3D inlet model is presented together with the converged solution of the developed coupled model (right side) and the full 3D CFD model. If we now compare these results with figure 9, it is shown that the presented models of the twin screw expander should be used depending on the accuracy target and available computational resources. This comparison is useful in the optimization process where depending on the optimization goal (optimization of the boundary conditions for the cycle analysis, optimization of the design using few port shape designs, optimization of a particular port shape by detailed analysis of the flow, etc) one of the following models can be used in order to make an optimization efficient.

\subsection{The pressure-volume diagram}

The most redeeming characteristic of the derived results is the shape of the pressure-volume diagram. P-V diagrams for the cases in table 1 are shown in figure 10 . In these figures, both the developed coupled model and the CFD model are presented.

As can be seen, the filling process which ideally should take place at approximately constant pressure, is in fact accompanied with high pressure losses. These pressure losses can be calculated due to the coupling between the thermodynamic multi-chamber model and the 3D inlet pipe model.

Results indicate that the biggest difference between the developed coupled model and the full CFD model is during the filling phase. However this difference is much smaller compared to the results when no inlet pipe pulsations are 


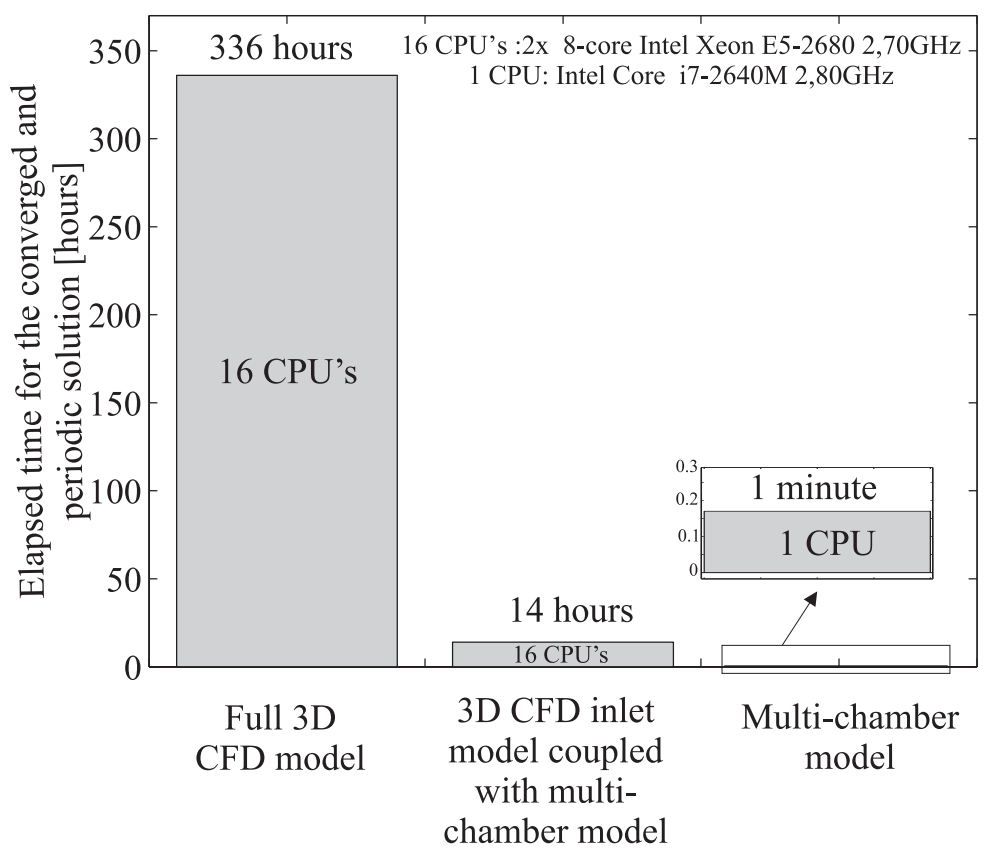

Figure 9: Total time and hardware requirements for converged solution for a full 3D CFD model, a coupled 3D inlet pipe model and multi-chamber model and a multi-chamber model

modelled (figure 8, left).

\subsection{Mass in the chamber and leakage flows}

The comparison of the mass in the chamber between the developed model and the CFD model is shown in figure 11. It can be seen that during the filling phase (until $\theta=126^{\circ}$ ) the mass in the chamber is changing rapidly. During the expansion, due to the leakage flows, the mass in the chamber will still change but not to such a large extent. Overall, the developed coupled model predicts the change in the mass in the chamber accurately compared to the full CFD model.

Mass flow rates through the tip and sealing leakage paths are shown in figure 12 . In the case of tip leakage flows (figure 12), both trailing and leading tip leakage flows are shown. The trailing tip is in fact the same as the leading tip, shifted by $\theta=90^{\circ}$ and with opposite sign.

Even though the trend of change in mass flow rates in clearance areas is close to the one calculated with the full CFD model, small differences still occur. Maximal differences between the results from a developed coupled model and a full 3D CFD model in figure $12 \mathrm{a}$ and figure $12 \mathrm{~b}$ are $0.005 \mathrm{~kg} / \mathrm{s}$ and $0.003 \mathrm{~kg} / \mathrm{s}$, respectively. This should be compared to the total mass flow rate of the expander which is for this case (pressure ratio 6 and $6000 \mathrm{rpm}$ ) $0.143 \mathrm{~kg} / \mathrm{s}$. The impact of these differences on the overall performance of the expander is negligible if we compare the influence of mass flow rates through all leakage paths within the twin screw expander. If all leakages are zero (not modeled or completely sealed), there would be an increase in power of $6.7 \%$ as presented in the next section. Obviously, the error in the coupled model will be much smaller than $6.7 \%$ as there is some misprediction of the leakages but no complete absence.

\subsection{Influence of clearance sealing}

In this section the influence of sealing clearance on the performance of the expander is presented. For this the expander with unsealed and completely sealed clearances was simulated (figure 13a). In the case of no leakage flows (completely sealed) the gain in power is around 6.7\% comparing to the unsealed expander. Here it should be noted that the clearances at the end planes are not simulated and thus their influence is not included. As opposed to compressors where the leakage flow (from higher pressure to lower) should be compressed again and therefore additional work 


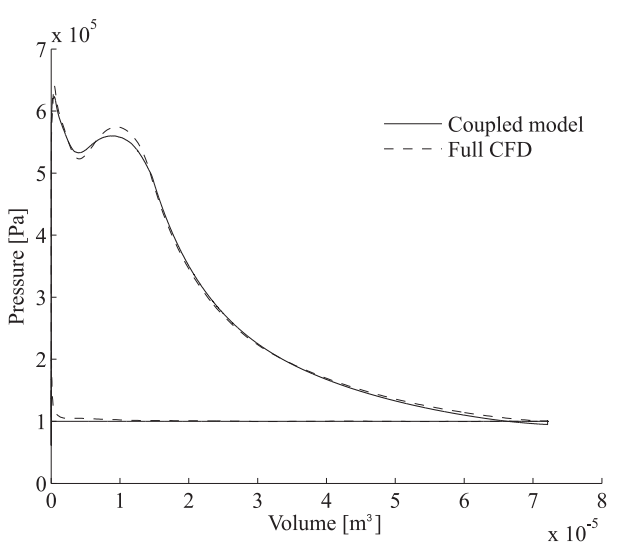

(a) Pressure ratio 6, $6000 \mathrm{rpm}$

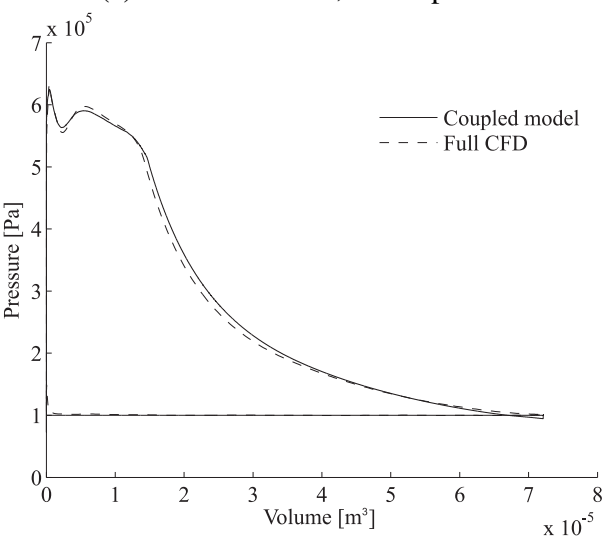

(c) Pressure ratio 6, $4000 \mathrm{rpm}$

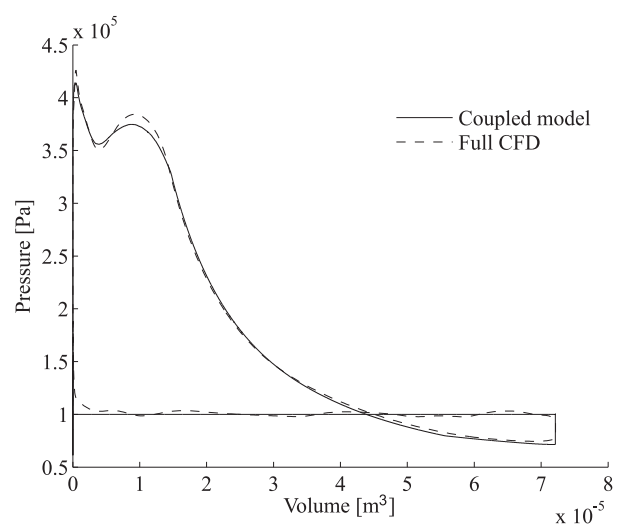

(b) Pressure ratio 4, 6000 rpm

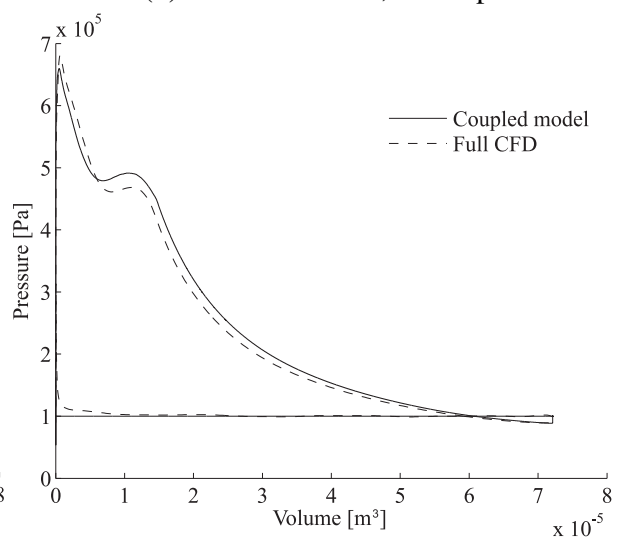

(d) Pressure ratio 6, $8000 \mathrm{rpm}$

Figure 10: Prediction of the coupled model compared with full CFD for the P-V diagram at different pressure ratios

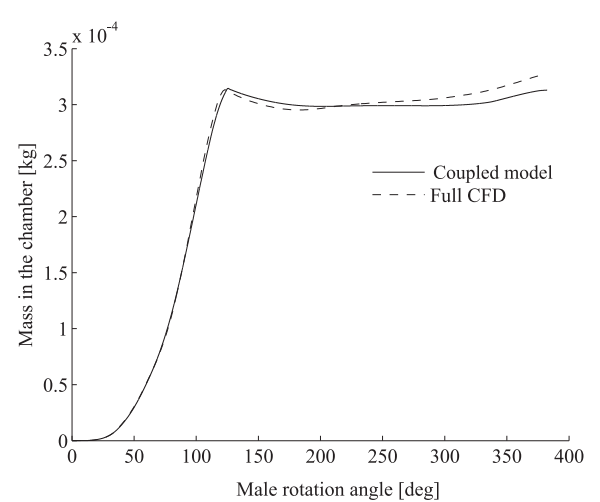

(a) Pressure ratio 6, $6000 \mathrm{rpm}$

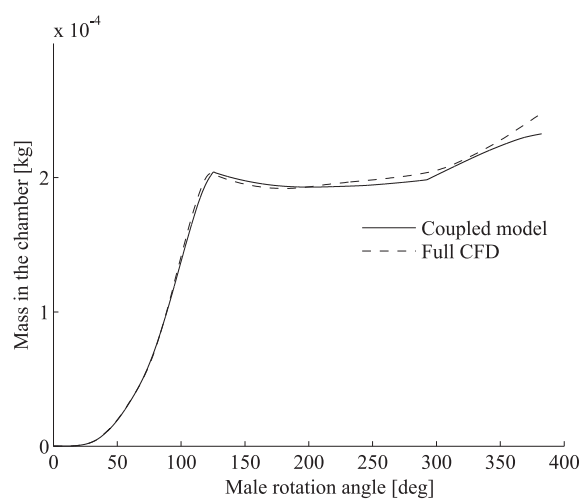

(b) Pressure ratio 4, $6000 \mathrm{rpm}$

Figure 11: Prediction of the coupled model compared with full CFD for the mass in a chamber at different pressure ratios

is used, in the case of an expander this leakage flow will be further expanded in the adjacent chamber which is on a lower pressure. As it can be seen in figure 13a, the mass in a chamber in the case of a completely sealed expander will stay constant while in the case of an unsealed expander it will change throughout the working cycle. 


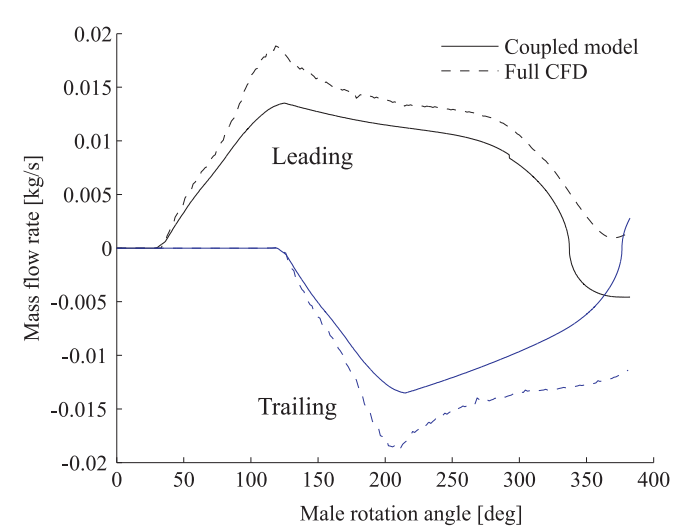

(a) Tip Leakages

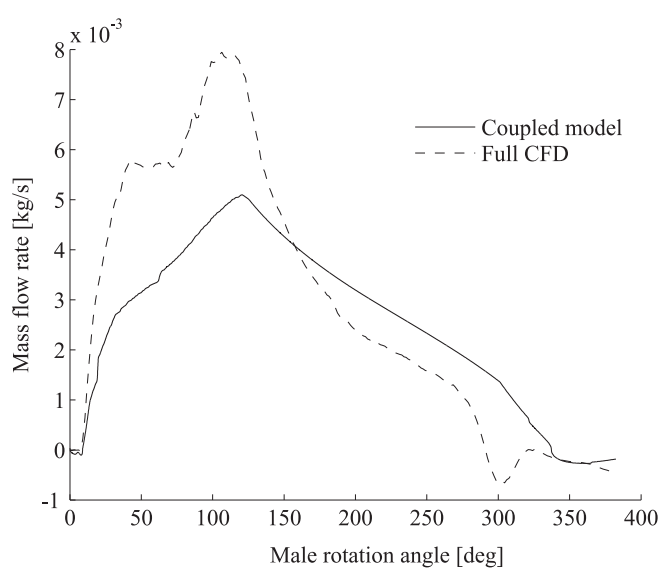

(b) Sealing leakage

Figure 12: Leakage flow through the tip and sealing clearance for pressure ratio 6 and rotational speed of 6000rpm

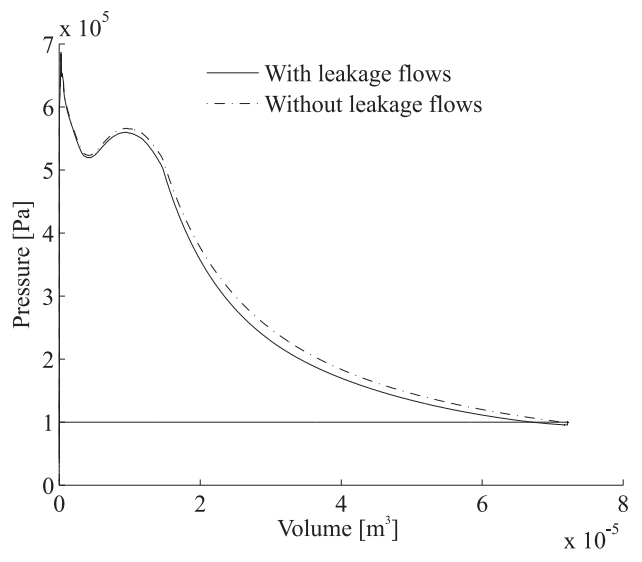

(a) P-V curve

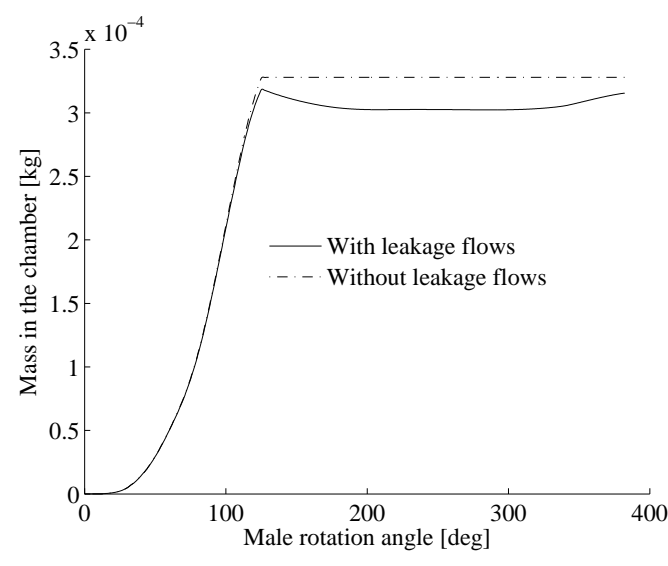

(b) Mass in the chamber

Figure 13: Influence of sealing clearance

\section{Conclusion}

This paper presents a mathematical model for the performance prediction of a twin screw expander. First the multichamber thermodynamic model is developed from the mass and energy conservation laws. Differential equations derived from these laws are solved together with the Aungier Redlich-Kwong Equation of State for R245fa in the rotational angle of the male rotor are employed in the model. Afterwards this model is coupled with a 3D inlet pipe model in order to better predict the pressure pulsations in the inlet pipe. This coupled model is validated with a full 3D CFD analysis of a twin screw expander recently presented by the authors.

The maximal deviation between full CFD and the presented coupled model is around 5\% for both power and mass flow rate. However, time and computational resources are significantly lower using the coupled model. Therefore it can be concluded that models presented and compared in this paper should be used depending on the accuracy target and available computational resources during the optimisation process. Also, this paper proved that the usage of CFD calculations can improve the capability of existing low-order models and this practice should be used more extensively in the future.

Additionally, the pressure pulsations in the inlet port affect not only the aeroacoustics in that domain but also the noise generated mechanically by rotor rattling. Therefore in the future the results of both models could be used 
for noise identification in screw expanders. This could be beneficial since the noise prediction has been an area of significant interest for a long time.

\section{Acknowledgement}

The results presented in this paper have been obtained within the frame of the IWT SBO-110006 project 'The Next Generation Organic Rankine Cycles' (www.orcnext.be), funded by the Institute for the Promotion and Innovation by Science and Technology in Flanders. This financial support is gratefully acknowledged.

\section{References}

[1] V. Lemort, L. Guillaume, A. Legros, S. Declaye, S. Quoilin, A comparison of piston, screw and scroll expanders for small scale Rankine cycle systems, Proceedings of the 3rd International Conference on Microgeneration and Related Technologies (2013).

[2] I. Smith, N. Stosic, A. Kovacevic, Power recovery from low grade heat by means of screw expanders, Woodhead Publishing in Mechanical Engineering, ISBN 978-1-78242-189-4 (2014).

[3] J. Hutker, A. Brümmer, Physics of a dry running unsynchronized twin screw expander, 8th International Conference on Compressors and their Systems, Woodhead Publishing, London (2013) 407-416.

4] N. Stosic, K. Hanjalic, A. Kovacevic, L. Milutinovic, Screw compressors mathematical modelling and performance calculation, Springer Berlin Heidelberg New York, ISBN-10 3-540-24275-9 (1988).

[5] J. S. Fleming, , Y. Tang, G. Cook, The twin helical screw compressor-Part 2: a mathematical model of the working process, Proceedings of the IMechE 212 (1998).

[6] F. Peveling, Ein Beitrag zur Optimierung adiabater Schraubenmachinen in Simulationsrechnungen, Doctoral Dissertation University of Dortmund (1987).

[7] M. Janicki, Modellierung und Simulation von Rotationsverdrangermaschinen, Doctoral Dissertation University of Dortmund (2007).

[8] I. H. Bell, Theoretical and Experimental Analysis of Liquid Flooded Compression in Scroll Compressors, Doctoral Thesis Purdue University (2011).

[9] N. Stosic, K. Hanjalic, Contribution towards modelling of two-stage reciprocating compressors, Int. J. Mech. Sci. 19 (1977) 439-445

320 [10] A. Huster, Untersuchung des instationären Füllvorgangs bei Schraubenmotoren, Doctoral Dissertation University Dortmund (1998).

[11] J. Vande Voorde, J. Vierendeels, E. Dick, Development of a Laplacian-based mesh generator for ALE calculations in rotary volumetric pumps and compressors, Computer Methods in Applied Mechanics and Engineering 193 (39-41) (2004) 4401-4415.

[12] A. Kovacevic, S. Rane, 3D CFD analysis of a twin screw expander, 8th International Conference on Compressors and their Systems (2013) 417-429.

[13] D. Margolis, Analytical Modeling of Helical Screw Turbines for Performance Prediction, ASME J. Engineering for Power 100 (1978) 482487.

[14] Z. Wang, Y. Zhang, Y. Sun, L. Wei, Numerical simulation and experimental study on the performance of screw expander, ASHRAE Transactions 116,part 2 (2010) 218-225.

[15] Y. Chen, N. Halm, E. Groll, J. Braun, Mathematical Modeling of Scroll Compressor. Part I-Compression Process Modeling, International Journal of Refrigeration 25 (2002) 731-750.

[16] B. Lee, T. Yanagisawa, M. Fukuta, S. Choi, A study on the leakage characteristics of tip seal mechanism in the scroll compressor, International Compressor Engineering Conference at Purdue University (2002).

[17] D. Kang, J. Kim, C. Sohn, Effects of leakage flow model on the thermodynamic performance of a scroll compressor, Int. Compressor Engineering Conf. at Purdue University (2002).

[18] I. H. Bell, E. Groll, J. Braun, W. Horton, A Computationally Efficient Hybrid Leakage Model for Modelling Leakage in Positive Displacement Compressors, Int. Compr. Engin. Conf. 1103 (2012)

[19] I. Papes, J. Degroote, J. Vierendeels, New insight in twin screw expander performance for small scale ORC systems by means of CFD analysis, Journal of Applied Thermal Engineering 90 (2015) 535-546.

[20] J. Sauls, S. Branch, Use of computational fluid dynamics to develop improved one-dimensional thermodynamic analyses of refrigerant screw compressors, 8th International Conference on Compressors and their Systems (2013) 591-600.

[21] I. Papes, J. Degroote, J. Vierendeels, Numerical simulation of a twin screw expander for performance prediction, IOP Conference Series Materials Science and Engineering 90(1):012059 (2015).

[22] J. Vande Voorde, J. Vierendeels, E. Dick, ALE Calculations of Flow Through Rotary Positive Displacement Machines, In Proc. of the ASME Fluids Engineering Divison Summer Meeting and Exhibition, FEDSM2005-77353, ISBN 0-7918-3760-2,Houston, USA (2005).

[23] M. J. Moran, H. N. Shapiro, D. D. Boettner, M. B. Bailey, Principles of Engineering Thermodynamics, 7th Edition, John Wiley \& Sons Ltd, (2011).

[24] R. H. Aungier, A fast, accurate real gas equation of state for fluid dynamic analysis applications, Journal of Fluids Engineering 117 (1995) 277-281. 